\title{
Magnetic trapping of buffer-gas cooled chromium atoms and prospects for the extension to paramagnetic molecules.
}

\author{
Joost M Bakker, ${ }^{1,2}$ Michael Stoll, ${ }^{2}$ Dennis R Weise, ${ }^{3}$ Oliver Vogelsang, ${ }^{3}$ Gerard Meijer, ${ }^{2}$ and Achim Peters ${ }^{1}$ \\ ${ }^{1}$ Humboldt Universität zu Berlin, Institut für Physik, Hausvogteiplatz 5-7,10117 Berlin, Germany \\ ${ }^{2}$ Fritz-Haber-Institut der Max-Planck-Gesellschaft, Faradayweg 4-6, D-14195 Berlin, Germany \\ ${ }^{3}$ Universität Konstanz, Fachbereich Physik, 78457 Konstanz, Germany
}

(Dated: November 1, 2018)

\begin{abstract}
We report the successful buffer-gas cooling and magnetic trapping of chromium atoms with densities exceeding $10^{12}$ atoms per $\mathrm{cm}^{3}$ at a temperature of $350 \mathrm{mK}$ for the trapped sample. The possibilities to extend the method to buffer-gas cool and magnetically trap molecules are discussed. To minimize the most important loss mechanism in magnetic trapping, molecules with a small spinspin interaction and a large rotational constant are preferred. Both the $\mathrm{CrH}\left({ }^{6} \Sigma^{+}\right.$ground state $)$ and $\mathrm{MnH}\left({ }^{7} \Sigma^{+}\right)$radicals appear to be suitable systems for future experiments.
\end{abstract}

\section{INTRODUCTION}

The buffer-gas cooling and magnetic trapping of $\mathrm{CaH}$ in 1998 constituted the first experiment where large samples of cold neutral molecules were confined by externally applied electromagnetic fields [1]. Since then, a number of alternative methods has been developed to produce and capture cold molecules, such as Stark deceleration of polar molecules in a molecular beam and subsequent electric trapping [2], photoassociation of laser-cooled atoms in a magneto-optical trap [3], and molecule formation by tuning through magnetic Feshbach resonances in an optical dipole trap [4]. Among these approaches, the thermalization with a cold buffer gas is the most general cooling method, as it is applicable to virtually all atomic and molecular species. It also offers the benefit of producing very large samples at sub-Kelvin temperatures, which form a good starting point for further cooling by other techniques, such as evaporative cooling.

Doyle and co-workers have successfully applied the buffer gas method [5] to trap atomic chromium and europium [6, 7] as well as several other atoms [8]. Apart from the above mentioned experiments on $\mathrm{CaH}$, they also demonstrated buffer-gas cooling of $\mathrm{VO}$ and $\mathrm{PbO}[9,10$ molecules. Although highly successful, buffer gas cooling in combination with magnetic trapping of neutral species up to now had only been implemented by this one group.

Here we report on our buffer-gas loading experiment, aiming at the production of large samples of trapped cold molecules. We have validated the performance of the new system by buffer-gas cooling and trapping of atomic chromium $(\mathrm{Cr})$. Cr was chosen as it has a large magnetic moment of $6 \mu_{B}$ and strong, well-characterized electronic transitions suitable for detection using absorption spectroscopy. Moreover, the most abundant isotope ${ }^{52} \mathrm{Cr}(86$ $\%$ ) has zero nuclear spin, which considerably simplifies the spectrum. As mentioned above, $\mathrm{Cr}$ was also one of the first atoms to be magnetically trapped using buffer-

*Electronic address: bakker@physik.hu-berlin.de gas cooling by Doyle and co-workers, and it has been shown that evaporative cooling to quantum degeneracy is feasible [11]. In our current experiments we trap $\mathrm{Cr}$ atoms and observe holding times exceeding one minute.

In the second part of the paper we describe the criteria we have used to select the molecules to be studied with this new experimental setup. We discuss the technological constraints as well as the relevant intrinsic properties of molecules that determine the chances of success of buffer-gas cooling experiments in conjunction with magnetic trapping.

\section{METHOD AND EXPERIMENTAL SETUP}

Buffer-gas cooling of atoms and molecules is done by thermalizing them with a cold background gas. Collisions with the background gas particles take out small quantities of energy of the particles to be cooled, and after many collisions the particles are thermalized with the background gas. The choice of background gas is restricted to those species that have at the (low) temperature of interest a large enough density to facilitate a large number of collisions while not reacting with the collision partner to form new species. Also, if buffer gas cooling is combined with trapping using external fields, the buffer gas should be insensitive to these fields. In practice, the only gas that fulfills these conditions is helium.

At the start of experiments, a dense helium vapor is created in an experimental cell. The buffer gas density is chosen such that hot particles injected into it are cooled sufficiently to be trapped by an inhomogeneous magnetic field before they can diffuse to the cell wall and adsorb to it. On the other hand, the density must be low enough to allow for the particles to reach the trap center. Optimal buffer gas densities are typically in the range of $10^{16}-10^{17}$ atoms per $\mathrm{cm}^{3}$, which corresponds to the vapor pressure of ${ }^{4} \mathrm{He}$ at $\sim 700 \mathrm{mK}$, or ${ }^{3} \mathrm{He}$ at $\sim 300 \mathrm{mK}[5]$.

After the creation of the required buffer gas density, the species to be cooled is injected into the cell. This can be done through capillary injection [12], or by ablation from a solid state precursor material placed within that 


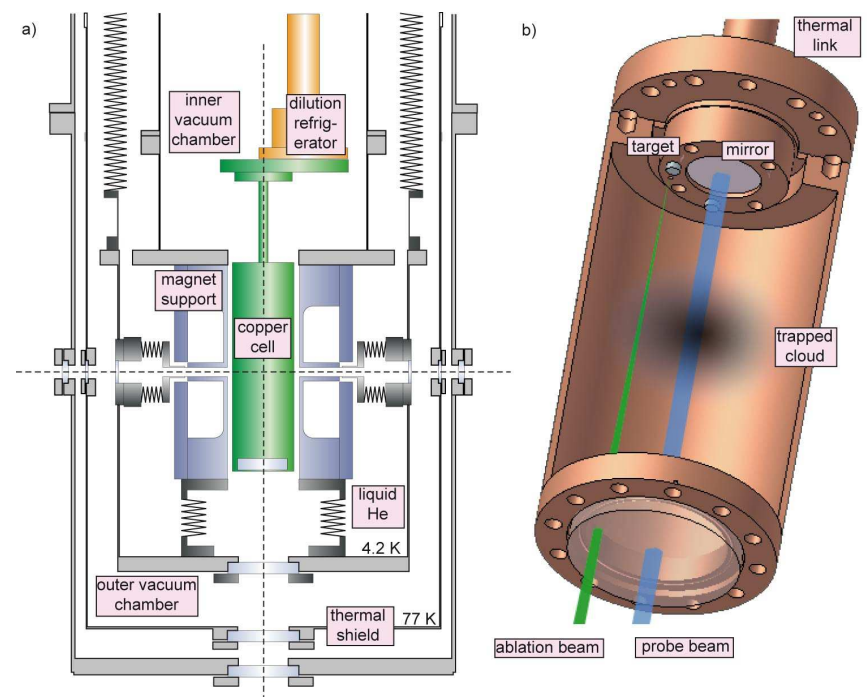

FIG. 1: Scheme of the experimental setup. Figure 19 shows the experimental cell within the custom-made cryostat, positioned within the magnet bore. Figure 10 depicts a cut through the copper cell itself.

cell. In another current project, efforts are made to use a molecular beam to inject molecules into the buffer-gas [13]. Upon injection into the cell, the particles collide with the helium atoms in the vapor. By thermalizing with the helium vapor, species in low-field seeking (LFS) states are drawn into the local magnetic field minimum; those in high-field seeking (HFS) states are repelled and lost at the cell wall. To fully isolate the trapped sample, it is necessary to remove the helium, as it forms a thermal link to the cell wall. This can be done by cooling the cell down, thereby effectively cryopumping the helium out.

For the cooled species to be magnetically confined, a (strong) magnetic field around the experimental cell, with a field minimum coinciding with the cell center, must be applied. This can be done using a magnetic quadrupole trap consisting of two parallel coils where counter-propagating currents create a zero magnetic field in the center between the coils. Typically, the trap depths required for buffer gas cooling should be on the order of a few Kelvin. As the trap depth $E$ (in units of temperature) is given by $E=\mu H / k_{B}$, where $\mu$ is the magnetic moment of the species $H$ the magnetic field magnitude, and $k_{B}$ the Boltzmann constant, this leads to typically required field strengths of $\sim 2 \mathrm{~T}$. These fields can be generated by coils of superconducting material, for which a cooling to liquid helium temperatures is required.

The refrigerator for cooling the experimental cell and the liquid helium bath that holds the magnet are implemented in a custom-made cryostat which is schematically depicted in Figure 1. The cryostat is built around a commercial ${ }^{3} \mathrm{He}-{ }^{4} \mathrm{He}$ dilution refrigerator system (Leiden Cryogenics Minikelvin 126-700), with a specified cooling power of $500 \mu \mathrm{W}$ at $120 \mathrm{mK}$. The magnet consists of two coils of $\mathrm{NbTi} / \mathrm{Cu}$ wire spun onto a titanium bobbin that is designed to withstand the repulsive forces of the two coils. The magnet design is similar to the Mark-3 design by Doyle and co-workers [14], but allows for three axes along which the cell can be optically interrogated: one vertical and two horizontal. From the bottom of the cryostat, three apertures at the different temperature stages of the cryostat (room temperature, $77 \mathrm{~K}$ and $4 \mathrm{~K}$ ) allow for the implementation of $75 \mathrm{~mm}$ diameter windows. From the top of the cryostat and from the four lateral sides there are apertures for $25 \mathrm{~mm}$ diameter windows. To be able to use the four side windows, there are $10 \mathrm{~mm}$ diameter clearances through the magnet support structure at the height of the trap center.

The experimental cell, made of oxygen-free high conductivity copper (OFHC), is attached to the mixing chamber of the dilution refrigerator and positioned in the center of the magnet bore. The cell is linked to the cryostat by a $10 \mathrm{~cm}$ long copper rod of $15 \mathrm{~mm}$ diameter forming a well-defined thermal impedance. Optical access into the cell is provided by a $50 \mathrm{~mm}$ diameter window sealing the bottom of the cell. A buffer gas fill line is implemented using a $\mathrm{CuNi}$ capillary. Finally, the cell is equipped with $\mathrm{RuO}$ thermistors and a resistive heater for temperature monitoring and control.

In the current experiment ${ }^{4} \mathrm{He}$ is used as a buffer gas and the appropriate density is created by bringing the entire cell to a temperature of choice. The density is limited by the pre-defined amount of helium let into the cell. Cr atoms are injected via laser-ablation from a solid chip of Cr, positioned at the top of the cell, using a 10 ns duration frequency-doubled Nd:YAG laser pulse at $532 \mathrm{~nm}$ with a typical pulse energy of $\sim 15 \mathrm{~mJ}$. We detect $\mathrm{Cr}$ atoms in their $m_{J}=+3$ sublevel of the ground state by monitoring absorption of light resonant with the $a^{7} S_{3}\left(m_{J}=+3\right) \leftarrow z^{7} P_{4}\left(m_{J}=+4\right)$ transition around $425 \mathrm{~nm}$. This is a nearly closed transition where almost all excited $\mathrm{Cr}$ atoms decay into the initial state (leakage $\left.\sim 10^{-6}\right)$. The light is generated by frequency doubling the output of a grating-stabilized diode laser (Toptica DL100) at $850 \mathrm{~nm}$ in a $\mathrm{KNbO}_{3}$ crystal within an external bow-tie cavity. The cavity is actively locked to the diode laser using a Pound-Drever-Hall scheme [15]. On long timescales the cavity length is adjusted piezo-electrically to match the laser frequency; on short time scales, the frequency of the diode laser is adjusted to match the cavity length by modulating the diode current. It must be stressed that the laser is not frequency-locked to an external reference, so that long-term frequency drifts can occur. The frequency-doubled light is amplitude stabilized by an acousto-optic modulator (AOM), spatially filtered, expanded and (optionally) sent through an aperture defining a beam diameter of a few $\mathrm{mm}$.

This beam is then sent into the cell using a beam splitter and retroreflected by a mirror mounted at the top of the cell. For the current experiments only the windows that give access from the bottom of the cell are implemented, and to minimize optical etalon effects all windows are wedged by $\sim 1^{\circ}$ and anti-reflection (AR) coated 
for the wavelengths used in the experiment. Light exiting the cell is directed onto a photomultiplier tube (PMT, Electron Tubes 9813QB). A fraction of the light that is not entering the cryostat is directed onto a second PMT (Electron Tubes 9814QB) for normalization and correction of residual intensity fluctuations. Typical light powers used are less than $1 \mu \mathrm{W}$, to avoid both heating of the cell and loss of atoms due to optical pumping effects. The measured transmission is thus obtained by normalizing the detection signal on the reference signal. When the detection laser is scanned in frequency, the signal is corrected for etalon effects by recording a background transmission signal on which the signal is normalized. In an alternative detection scheme, the transmitted beam is directly steered onto the light-sensitive chip of a CCD camera (PCO Imaging, Sensicam SuperVGA).

\section{MAGNETIC TRAPPING OF CHROMIUM}

Figure 2a depicts a typical recorded transmission trace spanning a time period of 1 minute. In this experiment, starting at $\mathrm{t}=-3.5 \mathrm{~s}$, the cell is heated for a period of $3 \mathrm{~s}$ with a heating power of $\sim 20 \mathrm{~mW}$. Then, at $\mathrm{t}=0 \mathrm{~s}$, the ablation laser $(15 \mathrm{~mJ})$ is fired to inject $\mathrm{Cr}$ atoms into the helium vapor. The optimal loading conditions are found in a procedure where the heating power and ablation are optimized to yield the best capture efficiencies. A field free measurement under the same conditions suggests that the heating over a few seconds ensures a full evaporation of the helium off the cell wall, whereas the short waiting period allows for the buffer gas to be cooled somewhat without noticeable cryopumping effects taking place. The $15 \mathrm{~mJ}$ used for the ablation is a compromise between signal intensity and the temperature at which the atoms are captured in the trap.

The trace shown in Figure $2 \mathrm{a}$ is recorded by repeatedly scanning the probe laser frequency over a range of $\sim 10 \mathrm{GHz}$ to obtain a magnetic-field broadened absorption line while minimizing the effect of long term drifts of the laser wavelength. In the scanned signal, some interesting dynamics can be observed. In the initial few seconds after ablation, the strong absorption signal first decreases, then shortly grows again and finally decays slowly. Additionally, a rapid narrowing of the line profile can be seen during the first 5 seconds following ablation. These different regimes will be discussed in detail below.

More detailed information about the density and temperature evolution of the trapped atom ensemble can be obtained by simulating the spectra and comparing them to the recorded ones. First we note that at temperatures below $1 \mathrm{~K}$ the expected Doppler broadening is less than $100 \mathrm{MHz}$, which is negligible in comparison with the observed Zeeman broadened linewidths of $1 \mathrm{GHz}$ and larger. For the modeling, it is assumed that the cloud of atoms is in thermal equilibrium and can be characterized by a Boltzmann temperature [16]. For any given point in the trapping field, the Zeeman shifted transi-

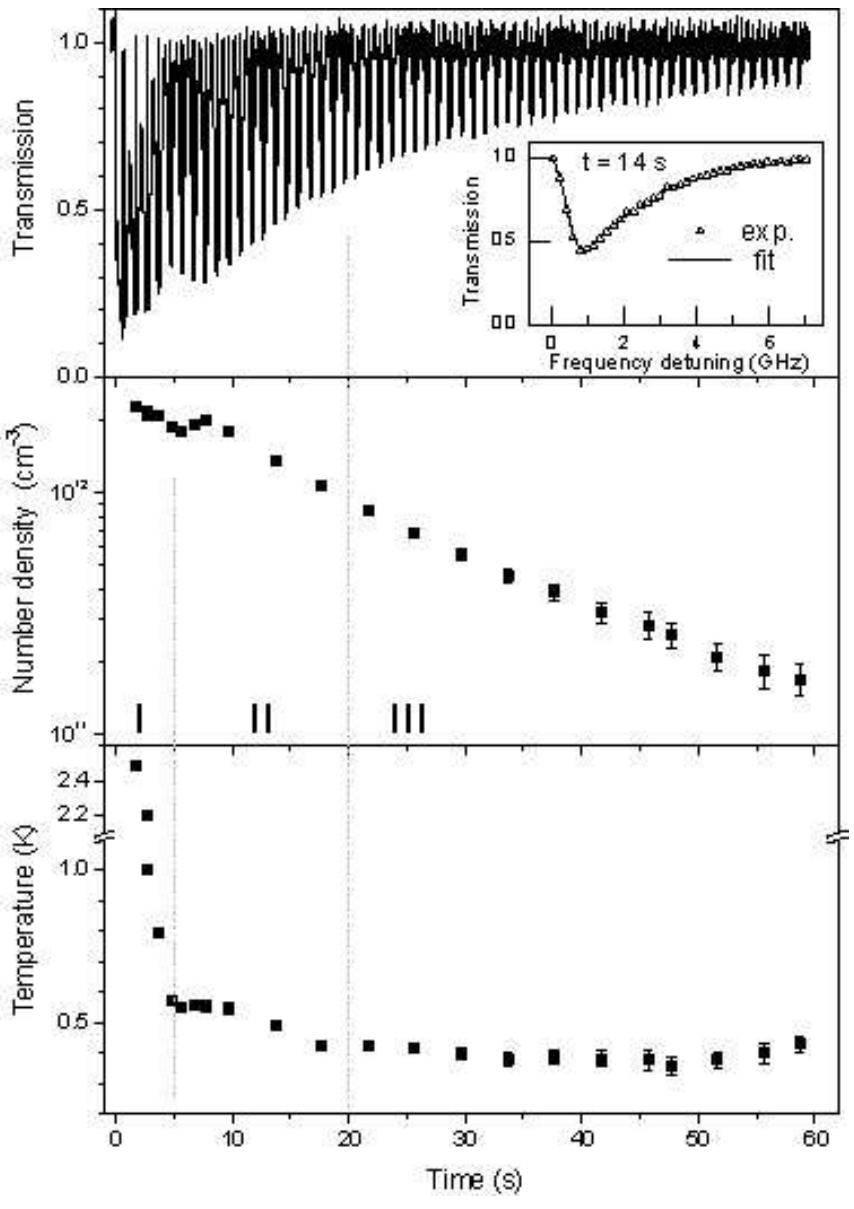

FIG. 2: Transmission through a sample of $\mathrm{Cr}$ atoms in the buffer gas cell (top graph). The probe laser frequency is scanned repeatedly over the atomic transition with a repetition rate of $1 \mathrm{~Hz}$. The inset shows a typical absorption profile (triangles) after $\sim 14 \mathrm{~s}$, together with a simulation (dotted line). The other two graphs show the evolution of the particle density (middle) and temperature (bottom graph) of the trapped atom ensemble over time.

tion frequency and a (relative) population density and a resulting absorption cross-section are calculated. A transmission spectrum is obtained by numerically integrating the absorption over the volume defined by the probe beam. From the inset in Figure $2 \mathrm{a}$ it can be seen that the simulated spectrum is in excellent agreement with the recorded data.

The simulations show that the spectral shape strongly depends on the probe beam parameters and we have thus carefully measured beam diameter and intensity profile. However, the relative position of the probe beam with respect to the center of the magnetic quadrupole trap is less amenable to direct measurements and must therefore be included in the model. This relative position is especially important when the probe beam is linearly polarized, as the projection of the driving $E$-field onto the quantization axis of the atoms, the inhomogeneous magnetic field, has a strong angular dependence. It is rather straightforward 
to derive that the angular dependence of the absorption coefficient is $\left(\cos ^{2} \theta+\sin ^{2} \theta \sin ^{2} \phi\right)$, where $\theta$ is the angle between magnetic field and light propagation axis, and $\phi$ that between polarization direction and magnetic field, respectively. To the best of our knowledge, this full angular dependence has not been implemented in modeling of data in previous buffer-gas loading experiments. Best agreement is obtained when it is assumed that our $2 \mathrm{~mm}$ diameter beam is offset by $0.3 \mathrm{~mm}$ from the trap center, which is well within the limits of optical alignment. The remaining free parameters used in the simulations are the particle density at the trap center and the Boltzmann temperature, which are found using a least-squares fitting procedure.

A possible error in the parameters obtained this way arises from a breakdown of the assumption of thermal equilibrium. This is likely the case at times shortly after the ablation pulse, where the changes in temperature are on comparable time scales as the time it takes to record one spectral profile. For this reason, the temperatures and densities obtained before $\mathrm{t}=5$ seconds are given without error bars and should be considered as indicative only. Additionally, long-term drifts in the laser frequency could amount to errors of a few \%. The densities and temperatures of the sample as obtained from the spectral simulations are depicted in the lower two graphs in Figure 2, The size of the error bars reflects the reduced signal-to-noise level at later times, when absorption signals become less pronounced.

Data at short times after ablation have not been published by Doyle and co-workers previously and it is interesting to speculate on the nature of the observed dynamics. The time evolution can roughly be divided into three periods which are indicated by I, II and III in Figure $2 \mathrm{~b}$. During period I, the temperature falls rapidly from $\sim 1.5 \mathrm{~K}$ at $\mathrm{t}=2 \mathrm{~s}$ to some $500 \mathrm{mK}$ after $5 \mathrm{~s}$. This is attributed to so-called spilling effects, where during trap loading the warmest particles are lost over the trap edge. As a result, the overall temperature drops.

In Period II, the sample temperature does not change as dramatically as in Period I. The sample appears to be in thermal equilibrium as it has a temperature similar to that of the cell wall. The obvious recurrence in the transmission spectrum after $5 \mathrm{~s}$ is also observed in the density. The increase in the density is interpreted as a net diffusion of cooled atoms into the trap center.

After $\sim 20 \mathrm{~s}$, the temperature of the cell wall (not shown) has dropped to around $350 \mathrm{mK}$ and the gas-phase helium within the cell is now cryopumped to the cell wall. As a consequence the sample now becomes thermally isolated and the temperature of the trapped cloud is now no longer decreasing. Its final temperature thus corresponds to the cell temperature at the time of thermal disconnect.

At times later than $20 \mathrm{~s}$, a clear continuous loss of $\mathrm{Cr}$ is observed. It is not expected that evaporation plays a large role at this point, as the effective trap depth at these temperatures is very large and evaporation rates are expected to decrease exponentially with effective trap

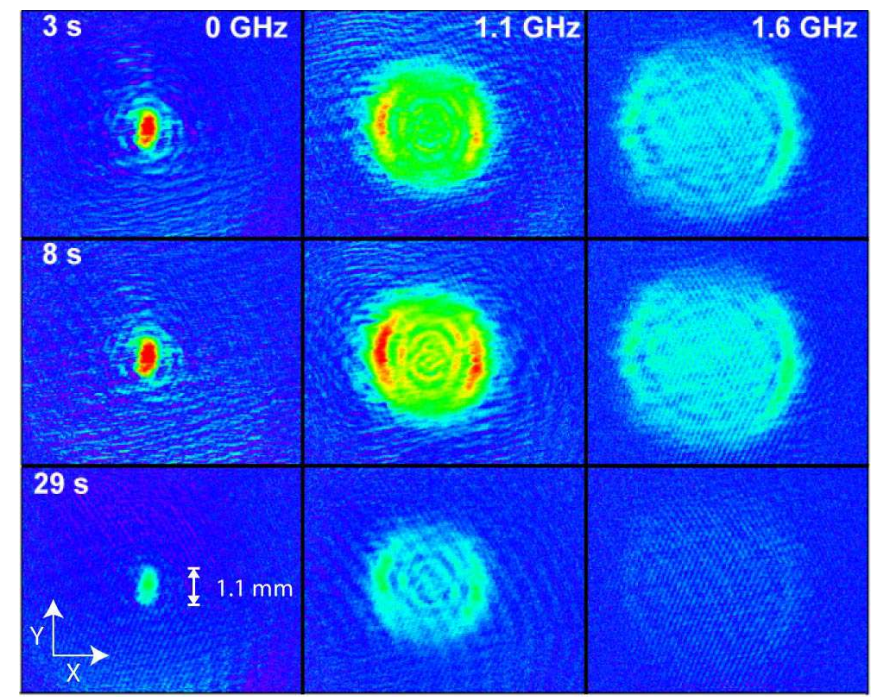

FIG. 3: Absorption images of a trapped cloud of $\mathrm{Cr}$ atoms, at three different times (columns) and at three different laser frequencies (rows). The size of the image is $8.6 \mathrm{~mm} \times 6.9 \mathrm{~mm}$.

depth [17]. A further indication for this is that the observed temperatures do not change, which rules out effective evaporative cooling. As additionally the buffer gas temperature is similar to or lower than the Cr temperature, we can thus rule out elastic collisions as a loss mechanism.

As a consequence, the observed trap loss must be due to inelastic collisions, which change the spin of the atom. Several such processes are known and they can be distinguished by the number of $\mathrm{Cr}$ atoms involved. The dominant loss mechanism may be found by fitting functional forms to the number density evolution. In an earlier set of data recorded over $180 \mathrm{~s}$ (where the signal was noisier and thus less suitable for quantitative data analysis) a clear signature is found of two-body loss processes, i.e. collisions in which two $\mathrm{Cr}$ atoms are involved. For the data presented in Figure 2, the signature of two body loss processes cannot be easily discerned from that due to collisions with background gas. If we leave one-body decay out of consideration, we obtain a maximum value for the two-body inelastic collision cross-section of $6 \cdot 10^{-13} \mathrm{~cm}^{2}$ at $\mathrm{T}=350 \mathrm{mK}$, consistent with a previous measurement [18.

In the measurements presented above, the probe laser diameter is limited to $\sim 2 \mathrm{~mm}$ and is aligned close to the trap center. This way not the whole trapping volume is addressed. In an alternative experimental configuration, the full waist of the collimated probe beam is sent through the trap center, retroreflected and steered directly onto the light-sensitive element of a CCD camera. For these imaging experiments the laser frequency is kept at a fixed value. Since the atomic resonance frequency is determined by the field-dependent Zeeman shift, a fixed frequency experiment selectively probes for atoms in specific 'shells' within the trapping volume. The results of 
these measurements are depicted in Figure 3 for three probe laser frequencies and three different times at the maximum trap depth of 6.8 Kelvin. The images shown (1 second gate time) are obtained as the ratio of an image recorded with $\mathrm{Cr}$ in the trap and one where no $\mathrm{Cr}$ is in the system. The size of the images is $8.6 \mathrm{~mm}$ (horizontal axis) by $6.9 \mathrm{~mm}$ (vertical). Since the beam is collimated, the spatial extent of the absorption signal on the CCD element has a 1:1 correspondence to the trapped cloud size.

The first set of images is recorded at the frequency of the field-free atomic transition. A strong absorption signal is clearly visible at the trap center. One can see that the absorption is anisotropic, which is a manifestation of the use of linearly polarized probe light. When the probe laser is tuned towards higher frequencies, a ring structure becomes apparent in the images. Here, it is obvious that the angular intensity distribution is proportional to $\sin ^{2} \phi$, as discussed above. At a frequencyoffset of $+1.1 \mathrm{GHz}$, the strongest absorption is observed in a ring with a radius of $\sim 1.5 \mathrm{~mm}$. The reason for such a pronounced ring is that the probe beam line-ofsight here coincides with a 'shell' of $\mathrm{Cr}$ atoms trapped at the same field magnitude. Again, the asymmetry attributed to the laser polarization is clearly visible. The ring structure allows for a consistency check of our trapping field calculations. If we assume that the probe beam is collimated well, this implies that at a radius of 1.5 $\mathrm{mm}$ the magnetic field calculated from the Zeeman shift $\Delta \nu=H \mu_{B}\left(g_{f} m_{J, f}-g_{i} m_{J, i}\right) / h$ is $76 \mathrm{mT}$. This is indeed consistent with our trapping field calculations $(69 \mathrm{mT}$ at $1.5 \mathrm{~mm}$ ), used in the spectral simulations described earlier.

Apart from this strong outer ring of absorption, some weaker but still pronounced rings are also visible. The origin of these rings is unknown to us. It is unlikely that they are due to population in lower $m_{J}$ states, as they persist for tens of seconds and it is expected that population in lower $m_{J}$ states is lost more quickly than in the $m_{J}=+3$ state due to a combination of lower trap depth and spin-exchange collisions. It can be speculated that they are caused by diffraction of the beam by the dense atomic cloud in the trap center. It is, however, unfortunately not straightforward to simulate this diffraction pattern due to our probe geometry where the probe beam passes the trapped cloud twice. Without diffractive effects included, a semi-quantitative agreement between our simulated data and the images is found. We therefore do not extract temperature and density information out of these images. For the absorption measurements where only a small probe beam is steered through the center of the cloud, such diffractive effects are not expected to play an important role as the cloud size is larger than the beamsize, and the sensitive area of the PMT is significantly larger than the probe beam diameter.

To investigate the potential of this system for future experiments on the buffer gas cooling and trapping of molecular species, it is of interest to evaluate the trap-

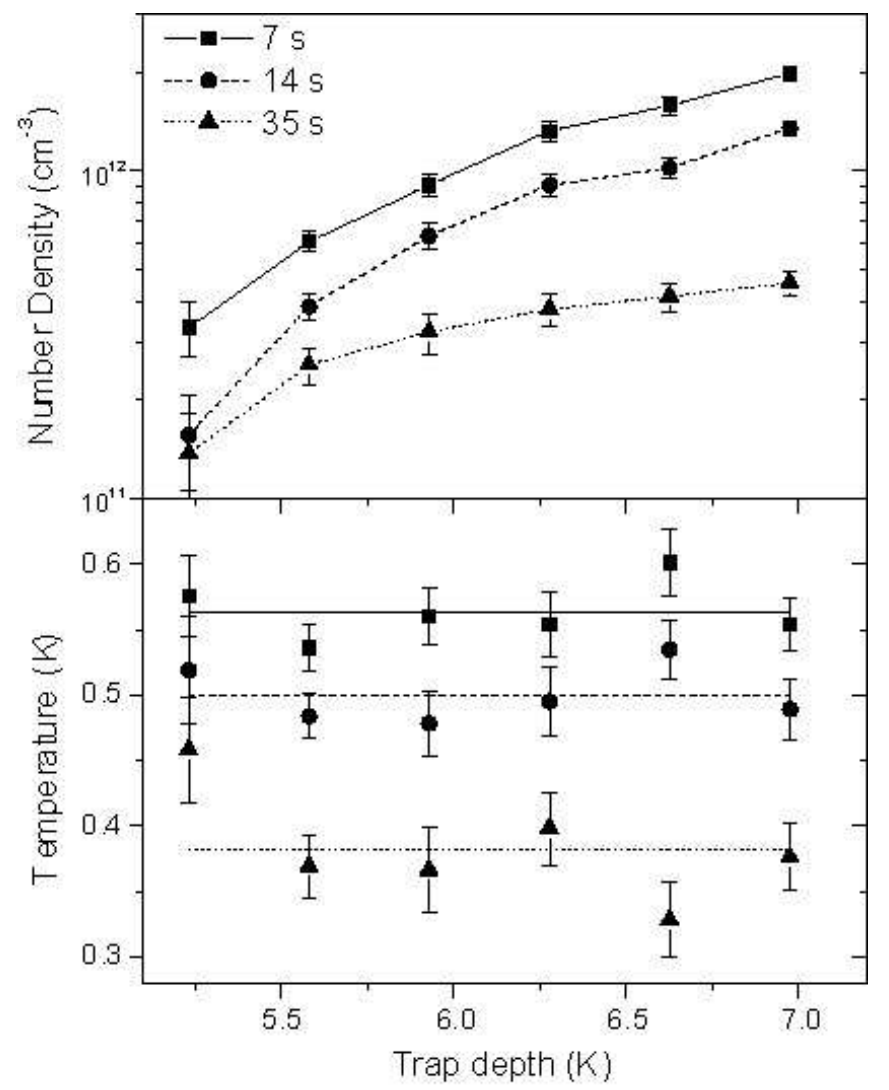

FIG. 4: Density (upper graph) and temperature (lower graph) of a trapped cloud of Cr atoms as a function of trap depth, measured at three different times; at $\mathrm{t}=7 \mathrm{~s}$ (squares), $\mathrm{t}=14$ $\mathrm{s}$ (circles) and $35 \mathrm{~s}$ (triangles) after ablation. The lines in the upper graph are guides to the eye, whereas in the bottom graph they represent the average temperature.

ping efficiencies at lower trap depths. Such a measurement is performed by lowering the current through the two anti-Helmholtz coils. The results of these tests are depicted in Figure 4 where the observed number densities and temperatures of a trapped $\mathrm{Cr}$ cloud, evaluated at three different times, are shown as a function of trap depth. Each value is obtained by averaging over several measurements. The trends that can be seen from these graphs are clear: a lower trap depth leads to a substantial drop in the number of captured atoms. The temperature of the trapped atoms is independent of trap depth. It seems thus obvious that a shallower trap captures less particles, while the temperature behavior gives us supporting evidence that no evaporative cooling, and thus no evaporation takes place. It is obvious from this Figure that if a molecule were to be trapped, it should possess a large enough magnetic moment to allow for a trap depth of at least $5 \mathrm{~K}$. Molecules that could fulfill this condition are discussed in Section IV 


\section{SEARCH FOR A MOLECULAR CANDIDATE FOR BUFFER GAS COOLING}

As He atoms are highly nonreactive and do not easily form new species, we can consider the helium buffer gas cooling a universal cooling method. As such, it has already been shown that one can use this technique as a source of cold molecules [19]. However, if the cooling is to be combined with magnetic trapping of the (paramagnetic) species, the universality is severely limited due to several constraints. At the heart of these constraints lie inelastic collisions, in which molecules in a low-field seeking (LFS) state undergo a transition into a high-field seeking (HFS) state, a process often referred to as Zeeman relaxation. The HFS molecules are then rapidly ejected from the trapping region. The ratio of inelastic to elastic collisions is thus crucial for the efficient transfer of energy while maintaining the molecules in a trappable state. The collision partners can be a molecule and a helium atom, but also two molecules. The former case is crucial for the feasibility of buffer-gas loading, while the latter governs a possible evaporative cooling process to further lower the temperature and increase the phasespace density of molecular samples.

Theoretical studies on the mechanisms of inelastic collisions with helium atoms in external fields are few, and have been limited to molecules in ${ }^{2} \Sigma$ and ${ }^{3} \Sigma$ ground states [20, 21, 22, 23, 24], although a study on collisions between $\mathrm{He}$ and the $\mathrm{OH}$ radical (in a ${ }^{2} \Pi$ state) is forthcoming [25]. For ${ }^{2} \Sigma$ molecules the Zeeman relaxation occurs through spin-rotational coupling between the rotational ground state and rotationally excited states [26]. Calculations for $\mathrm{CaF}$ and $\mathrm{CaH}$ molecules have shown that Zeeman relaxation rates are strongly dependent on the spin-rotation interaction constant $\gamma_{s r}$ and decrease with the energy difference between rotational states. The experimental data available for these molecules confirm this [1, 27].

For higher-spin states, no experimental data is available. It is, however, predicted that the Zeeman relaxation rates are considerably higher. In ${ }^{3} \Sigma$ states, the spin-spin interaction is the dominant factor and again a larger energy difference between the rotational states decreases the couplings between LFS and HFS states. As a consequence, the spin-spin interaction parameter $\lambda_{s s}$ should be relatively small and the rotational constant large to successfully trap molecules [26]. For the $\mathrm{NH}\left({ }^{3} \Sigma^{-}\right)$radical, the calculated ratio of elastic to inelastic collisions has been shown to be favorable for trapping experiments [24].

About the collisional properties of molecules in ground states other than ${ }^{2} \Sigma$ or ${ }^{3} \Sigma$ little is known. An unsuccessful attempt was made to buffer gas cool and trap VO molecules $\left({ }^{4} \Sigma^{-}\right.$ground state), but it is unclear whether Zeeman relaxation was a problem [9]. Theoretical calculations that predict collisional properties of such highspin molecules are unknown to us. Based on the lack of such data, we here propose to buffer gas cool molecules
TABLE I: Diatomic molecules with high-spin electronic ground states and their rotational constant (B), spin-spin interaction constant $\left(\lambda_{s s}\right)$ and spin-rotation interaction constant $\left(\gamma_{s r}\right)$. All numbers are given in $\mathrm{cm}^{-1}$. The ratio of $\lambda_{s s}$ over $\mathrm{B}$ is an indicator for the strength of Zeeman relaxation.

\begin{tabular}{|c|c|c|c|c|}
\hline & $B$ & $\lambda_{s s}$ & $\left|\lambda_{s s} / B\right|$ & $\begin{array}{r}\gamma_{s r} \text { ref. } \\
\times 10^{3}\end{array}$ \\
\hline $\mathrm{GdO}{ }^{9} \Sigma^{-}$ & 0.355 & -0.104 & 0.29 & $0.1[2$ \\
\hline $\mathrm{MnH}{ }^{7} \Sigma^{+}$ & 5.606 & -0.004 & $7.210^{-4}$ & $31.3[2$ \\
\hline $\mathrm{MnF}{ }^{7} \Sigma^{+}$ & 0.353 & -0.005 & 1.310 & $0.6[30$ \\
\hline $\mathrm{MnCl}{ }^{7} \Sigma^{+}$ & 0.158 & 0.037 & 0.24 & $0.4[31]$ \\
\hline $\mathrm{CrH}{ }^{6} \Sigma^{+}$ & 6.132 & 0.233 & $3.810^{-2}$ & $50.3[32]$ \\
\hline $\mathrm{MnO}{ }^{6} \Sigma^{+}$ & 0.501 & 0.574 & 1.1 & $-2.4[33]$ \\
\hline $\mathrm{CrF}$ & 0.379 & 0.539 & 1.4 & $13.6[34]$ \\
\hline $\operatorname{MnS}{ }^{6} \Sigma^{+}$ & 0.195 & 0.350 & 1.8 & $-2.4[35]$ \\
\hline $\mathrm{CrCl}{ }^{6} \Sigma^{+}$ & 0.167 & 0.266 & 1.6 & $2.2 \quad[34]$ \\
\hline $\mathrm{CrO}{ }^{5} \Pi_{r}$ & 0. & 1.148 & 2.2 & $10.5[36]$ \\
\hline $\mathrm{CrN}$ & 0.624 & 2.611 & 4.2 & $7.0[36]$ \\
\hline $\mathrm{VO}$ & 0.546 & 2.031 & 3.7 & 22.5 [37] \\
\hline $\mathrm{NbO}{ }^{4} \Sigma^{-}$ & 0.432 & 15.58 & 36 & 334.0 [38] \\
\hline $\mathrm{TiH}$ & 5.362 & & & $182.3[39]$ \\
\hline$\overline{3} \Sigma^{-}$ & 16.343 & 0.920 & $5.610^{-2}$ & $54.7[40]$ \\
\hline $\mathrm{O}_{2}$ & 1.438 & 1.985 & 1.4 & $-8.4[41]$ \\
\hline $\mathrm{CaH}{ }^{2} \Sigma^{+}$ & 4.229 & & & $46.7 \quad[42]$ \\
\hline $\mathrm{CaF}^{2}{ }^{2} \Sigma^{+}$ & 0.339 & & & $1.3[27$ \\
\hline
\end{tabular}

with a high-spin ground state to investigate their suitability for magnetic trapping and possible evaporative cooling experiments. By extrapolating the cases built for ${ }^{2} \Sigma$ and ${ }^{3} \Sigma$ molecules, we assume that the candidate molecule should possess a large rotational constant B and a small spin-spin interaction constant $\lambda_{s s}$ to minimize spin-spin couplings. As diatomics containing hydrogen atoms have the largest rotational constants, metal hydrides are a natural choice.

Apart from such general criteria more practical considerations also play a role. As is observed above, the trap depth plays a crucial role in the possibility to confine species over longer time periods. In the present experiment, trap depths of $5.3 \mathrm{~K}$ and above have been employed with success. A useful quantity to describe trapping is the capture efficiency parameter $\eta$, defined as the ratio of trap depth to sample temperature; $\eta=\mu H_{\max } / k_{B} T_{\text {mol }}$, where $\mu$ is the molecular magnetic moment, $H_{\max }$ the magnetic field strength at the trap edge and $T_{m o l}$ the Boltzmann temperature of the molecular sample. In the current experiment the trap edge is formed by the cell wall, where the highest achievable magnetic field is 1.7 $\mathrm{T}$. Thus, at the observed initial temperatures of $1.4 \mathrm{~K}$ (evaluated at $\mathrm{t}=2 \mathrm{~s}), \eta$ should be larger than 3.8 for a successful (initial) confinement of a substantial sample of molecules. This yields a minimum magnetic moment of about $4.5 \mu_{B}$.

Based on the above mentioned criteria, we have per- 
formed an extensive literature search to find an interesting and suitable molecular candidate for buffer gas cooling and magnetic trapping. The results are collected in Table I1. To compile this table, only molecules have been listed that possess an electronic ground state with at least a fourfold spin multiplicity. For comparison, the molecules studied so far using buffer-gas cooling have been included: $\mathrm{CaH}, \mathrm{NH}, \mathrm{CaF}$ as well as the oxygen molecule $\left({ }^{16} \mathrm{O}^{18} \mathrm{O}\right)$, a long time prime candidate. As a further restriction, only molecules that have been observed in experimental work and for which an experimental value of the rotational constant is available are included. A column is added with the ratio of the spin-spin interaction and rotational constants, $\left|\lambda_{s s} / B\right|$, to guide the selection process in minimizing the expected effects of Zeeman relaxation.

An inspection of this table indicates that there are three molecules that clearly stand out in terms of expected stability against Zeeman relaxation: $\mathrm{CrH}$ in its ${ }^{6} \Sigma^{+}$ground state, $\mathrm{MnH}\left({ }^{7} \Sigma^{+}\right)$and $\mathrm{MnF}\left({ }^{7} \Sigma^{+}\right)$. All three molecules possess a large magnetic moment, which is nominally 5 and $6 \mu_{B}$, respectively. We disregard the MnF molecule, as it has a substantially smaller rotational constant, which would result in level crossings between different rotational manifolds at fields lower than $0.25 \mathrm{~T}$ [54]. One could point out that the TiH molecule also possesses a large magnetic moment and rotational constant that should avoid these level crossings; however, it is believed that non- $\Sigma$ state molecules are highly susceptible to Zeeman relaxation, as direct couplings between Zeeman states tend to become large [25]. Since in addition no value of the spin-spin interaction constant could be found for $\mathrm{TiH}$, it is left out of consideration.

Both $\mathrm{CrH}$ and $\mathrm{MnH}$ fulfill the requirements of a large rotational constant, a small spin-spin interaction constant while at the same time having a high-spin ground state which should be amenable to magnetic trapping. Both $\mathrm{CrH}$ and $\mathrm{MnH}$ were first observed in the 1930's by Gaydon and Pearse 43, 44]. CrH has by far attracted the most experimental attention as a prototype of a high-spin diatomic molecule, especially since it was discovered in the emission spectra of L-type brown dwarfs [45]; for an overview, see [46] and references therein. Because of its high-spin ground state, $\mathrm{CrH}$ is employed as a probe for magnetic field strengths in sunspots [47]. The magnetic parameters of $\mathrm{CrH}$ are well-established in the $\mathrm{X}^{6} \Sigma^{+}$electronic ground state by FIR laser-magnetic resonance spectroscopy [32]. Recently, we also measured the Zeeman spectrum of the $\mathrm{A}^{6} \Sigma^{+} \leftarrow \mathrm{X}^{6} \Sigma^{+}(1-0)$ transition around $767 \mathrm{~nm}$, thereby determining the spectroscopic parameters of the excited state that are needed to simulate spectra recorded in an inhomogeneous magnetic field [48]. For $\mathrm{MnH}$, the experimental studies are more sparse, which is likely due to the highly complex structure of the spectroscopic transitions which are split into numerous hyperfine components. The $\mathrm{A}^{7} \Pi^{+} \leftarrow \mathrm{X}^{7} \Sigma^{+}(0-0)$ and (10) transitions near 624 and $568 \mathrm{~nm}$, respectively, are well characterized [49, 50, 51] and the ground state has been studied using ESR spectroscopy [52, 53].

Based on the extensive experimental information available, it seems that the $\mathrm{CrH}$ radical is currently the most promising high-spin candidate for studying the mechanisms involved in buffer gas cooling, although it can not be ruled out that the significantly lower spin-spin interaction of $\mathrm{MnH}$ will, in the end, favour this molecule.

\section{CONCLUSIONS}

We have demonstrated buffer-gas loading of paramagnetic species into a magnetic trap. In first experiments we confined Cr atoms with storage times of more than one minute. The use of an absorption based detection scheme combined with a simulation of the absorption properties of the cloud in the presence of the inhomogeneous magnetic trapping field allows us to extract relevant parameters. Given storage times of more than one minute, thermal contact between the trapped cloud and the walls of the experimental cell is reliably broken after a period of $20 \mathrm{~s}$. The final temperatures of $\sim 350 \mathrm{mK}$ reached are determined by the temperature of the buffer gas at thermal disconnect. Observed initial number densities exceed $10^{12}$ per $\mathrm{cm}^{3}$. We have also succeeded in implementing continuous non-destructive absorption imaging of the trapped cloud, which can provide complementary information on temperature and internal state distribution over the whole trapping volume.

Progressing towards the preparation of cold molecules, we have outlined the criteria for selecting a molecular candidate to study in buffer gas cooling experiments. Such a molecule should have, apart from a high magnetic moment, a low cross-section for spin-changing collisions which would result in trap loss. Due to their large rotational constants and small spin-spin interaction coefficients, the $\mathrm{MnH}$ and $\mathrm{CrH}$ molecules have been identified as potentially suitable species. We will thus proceed by experimentally investigating the relevant collisional properties of these molecules. Measurements of the effect of trap depth on the capture efficiency show that, if the spin-changing collision rates are sufficiently low, the current experimental setup should allow for trapping and thermally isolating $\mathrm{CrH}$ and $\mathrm{MnH}$.

\section{Acknowledgment}

We gratefully acknowledge the support by the Deutsche Forschungs Gemeinschaft (Forschergruppe Quantengase FOR282/2-1TP8). JMB acknowledges funding through the European Network for Cold Molecules (RTN2-2001-00498). We thank Bretislav Friedrich and John M. Doyle for stimulating discussions and their help in constructing this experiment. Last but not least we want to thank Tim Steimle for bringing $\mathrm{MnH}$ to our attention, and the referee for helpful comments that considerably improved the manuscript. 
[1] J. D. Weinstein, R. deCarvalho, T. Guillet, B. Friedrich, and J. M. Doyle, Nature 395, 148 (1998).

[2] H. L. Bethlem, G. Berden, F. M. H. Crompvoets, R. T. Jongma, A. J. A. van Roij, and G. Meijer, Nature 406, 491 (2000).

[3] N. Vanhaecke, W. de Souza Melo, B. L. Tolra, D. Comparat, and P. Pillet, Phys. Rev. Lett. 89, 063001 (2002).

[4] S. Jochim, M. Bartenstein, A. Altmeyer, G. Hendl, C. Chin, J. H. Denschlag, and R. Grimm, Phys. Rev. Lett. 91, 240402 (2003).

[5] R. deCarvalho, J. M. Doyle, B. Friedrich, T. Guillet, J. Kim, D. Patterson, and J. D. Weinstein, Eur. Phys. J. D 7, 289 (1999).

[6] J. D. Weinstein, R. deCarvalho, J. Kim, D. Patterson, B. Friedrich, and J. M. Doyle, Phys. Rev. A 57, R3173 (1998).

[7] J. Kim, B. Friedrich, D. P. Katz, D. Patterson, J. D. Weinstein, R. deCarvalho, and J. M. Doyle, Phys. Rev. Lett. 78, 3665 (1997).

[8] C. I. Hancox, S. C. Doret, M. T. Hummon, L. J. Luo, and J. M. Doyle, Nature 431, 281 (2004).

[9] J. D. Weinstein, R. deCarvalho, K. Amar, A. Boca, B. C. Odom, B. Friedrich, and J. M. Doyle, J. Chem. Phys. 109, 2656 (1998).

[10] D. Egorov, J. D. Weinstein, D. Patterson, B. Friedrich, and J. M. Doyle, Phys. Rev. A 63, 030501 (2001).

[11] A. Griesmaier, J. Werner, S. Hensler, J. Stuhler, and T. Pfau, Phys. Rev. Lett. 94, 160401 (2005).

[12] J. K. Messer and F. C. Delucia, Phys. Rev. Lett. 53, 2555 (1984).

[13] D. Egorov, W. C. Campbell, B. Friedrich, S. E. Maxwell, E. Tsikata, L. D. van Buuren, and J. M. Doyle, Eur. Phys. J. D 31, 307 (2004).

[14] J. G. E. Harris, R. A. Michniak, S. V. Nguyen, W. C. Campbell, D. Egorov, S. E. Maxwell, L. D. van Buuren, and J. M. Doyle, Rev. Sci. Instrum. 75, 17 (2004).

[15] R. W. P. Drever, J. L. Hall, F. V. Kowalski, J. Hough, G. M. Ford, A. J. Munley, and H. Ward, Appl. Phys. B 31, 97 (1983).

[16] O. J. Luiten, M. W. Reynolds, and J. T. M. Walraven, Phys. Rev. A 53, 381 (1996).

[17] W. Ketterle and N. J. van Druten, Adv. Atom. Mol. Opt. Phys. 37, 181 (1996).

[18] R. deCarvalho, C. I. Hancox, and J. M. Doyle, J. Opt. Soc. Am. B 20, 1131 (2003).

[19] S. E. Maxwell, N. Brahms, R. deCarvalho, D. R. Glenn, J. S. Helton, S. V. Nguyen, D. Patterson, J. Petricka, D. DeMille, and J. M. Doyle, Phys. Rev. Lett. 95, 173201 (2005).

[20] A. Volpi and J. L. Bohn, Physical Review A 65, 052712 (2002).

[21] R. V. Krems, A. Dalgarno, N. Balakrishnan, and G. C. Groenenboom, Phys. Rev. A 67, 060703 (2003).

[22] R. V. Krems, H. R. Sadeghpour, A. Dalgarno, D. Zgid, J. Klos, and G. Chalasinski, Phys. Rev. A 68, 051401 (2003).

[23] R. Krems and A. Dalgarno, J. Chem. Phys. 117, 118 (2002).

[24] H. Cybulski, R. V. Krems, H. R. Sadeghpour, A. Dalgarno, J. Klos, G. C. Groenenboom, A. van der Avoird, D. Zgid, and G. Chalasinski, J. Chem. Phys. 122, 094307
(2005).

[25] G. Groenenboom, private communications.

[26] R. V. Krems and A. Dalgarno, J. Chem. Phys. 120, 2296 (2004).

[27] K. Maussang, D. Egorov, J. S. Helton, S. V. Nguyen, and J. M. Doyle, Phys. Rev. Lett. 94, 123002 (2005).

[28] L. A. Kaledin, M. G. Erickson, and M. C. Heaven, J. Mol. Spec. 165, 323 (1994).

[29] I. E. Gordon, D. R. T. Appadoo, A. Shayesteh, K. A. Walker, and P. F. Bernath, J. Mol. Spec. 229, 145 (2005).

[30] P. M. Sheridan and L. M. Ziurys, Chem. Phys. Lett. 380, 632 (2003).

[31] D. T. Halfen and L. M. Ziurys, J. Chem. Phys. 122, 054309 (2005).

[32] S. M. Corkery, J. M. Brown, S. P. Beaton, and K. M. Evenson, J. Mol. Spec. 149, 257 (1991).

[33] K. Namiki and S. Saito, J. Chem. Phys. 107, 8848 (1997).

[34] K. Katoh, T. Okabayashi, M. Tanimoto, Y. Sumiyoshi, and Y. Endo, J. Chem. Phys. 120, 7927 (2004).

[35] J. M. Thompsen, M. A. Brewster, and L. M. Ziurys, J. Chem. Phys. 116, 10212 (2002).

[36] P. M. Sheridan, M. A. Brewster, and L. M. Ziurys, Astrophys. J. 576, 1108 (2002).

[37] A. S. C. Cheung, R. C. Hansen, and A. J. Merer, J. Mol. Spec. 91, 165 (1982).

[38] A. G. Adam, Y. Azuma, J. A. Barry, A. J. Merer, U. Sassenberg, J. O. Schroder, G. Cheval, and J. L. Femenias, J. Chem. Phys. 100, 6240 (1994).

[39] O. Launila and B. Lindgren, J. Chem. Phys. 104, 6418 (1996).

[40] C. R. Brazier, R. S. Ram, and P. F. Bernath, J. Mol. Spec. 120, 381 (1986).

[41] G. Berden, R. Engeln, P. C. M. Christianen, J. C. Maan, and G. Meijer, Phys. Rev. A 58, 3114 (1998).

[42] W. L. Barclay, M. A. Anderson, and L. M. Ziurys, Astrophys. J. 408, L65 (1993).

[43] A. G. Gaydon and R. W. B. Pearse, Nature 140, 110 (1937).

[44] R. W. B. Pearse and A. G. Gaydon, Proc. Phys. Soc. 50, 201 (1938).

[45] J. D. Kirkpatrick, I. N. Reid, J. Liebert, R. M. Cutri, B. Nelson, C. A. Beichman, C. C. Dahn, D. G. Monet, J. E. Gizis, and M. F. Skrutskie, Astrophys. J. 519, 802 (1999).

[46] S. Shin, D. J. Brugh, and M. D. Morse, Astrophys. J. 619, 407 (2005)

[47] O. Engvold, H. Wöhl, and J. Brault, Astron.Astrophys.Suppl.Series 42, 209 (1980).

[48] J. Chen, J. M. Bakker, A. Peters, M. Stoll, G. Meijer, and T. C. Steimle, to be published (????).

[49] T. D. Varberg, R. W. Field, and A. J. Merer, J. Chem. Phys. 92, 7123 (1990).

[50] T. D. Varberg, R. W. Field, and A. J. Merer, J. Chem. Phys. 95, 1563 (1991).

[51] T. D. Varberg, J. A. Gray, R. W. Field, and A. J. Merer, J. Mol. Spec. 156, 296 (1992).

[52] R. J. Vanzee, D. A. Garland, and W. Weltner, J. Chem. Phys. 84, 5968 (1986).

[53] R. J. Vanzee, D. A. Garland, and W. Weltner, J. Chem. Phys. 85, 3237 (1986).

[54] The magnetic field at which the first rotational level 
crossings can occur can be approximated by $H_{\text {cross }}=$ $2 B / \mu$ 
This figure "cr-trapdepth-variation.jpg" is available in "jpg" format from: http://arxiv.org/ps/physics/0604079v3 
This figure "cr-trapped.jpg" is available in "jpg" format from: http://arxiv.org/ps/physics/0604079v3 